\title{
Analytic Treatment for Electrical MHD Non-Newtonian Fluid Flow over a Stretching Sheet through a Porous Medium
}

\author{
Gossaye Aliy Adem (iD \\ Department of Mathematics, University of Gondar, Ethiopia \\ Correspondence should be addressed to Gossaye Aliy Adem; gosyy610@gmail.com
}

Received 25 August 2020; Revised 26 November 2020; Accepted 11 December 2020; Published 28 December 2020

Academic Editor: John D. Clayton

Copyright (C) 2020 Gossaye Aliy Adem. This is an open access article distributed under the Creative Commons Attribution License, which permits unrestricted use, distribution, and reproduction in any medium, provided the original work is properly cited.

\begin{abstract}
In this study, an attempt has been made to investigate the mass and heat transfer effects in a BLF through a porous medium of an electrically conducting viscoelastic fluid subject to a transverse magnetic field in the existence of an external electric field, heat source/sink, and chemical reaction. It has been considered the effects of the electric field, viscous and Joule dissipations, radiation, and internal heat generation/absorption. Closed-form solutions for the boundary layer equations of viscoelastic, second-grade, and Walters' $\mathrm{B}^{\prime}$ fluid models are considered. The method of the solution includes similarity transformation. The converted equations of thermal and mass transport are calculated using the optimal homotopy asymptotic method (OHAM). The solutions of the temperature field for both prescribed surface temperature (PST) and prescribed surface heat flux (PHF) are found. It is vital to remark that the interaction of the magnetic field is found to be counterproductive in enhancing velocity and concentration distribution, whereas the presence of chemical reaction, as well as a porous matrix with moderate values of the magnetic parameter, reduces the temperature and concentration fields at all points of the flow domain.
\end{abstract}

\section{Introduction}

The significance of fluid flow over a stretching surface can be professed for its ever-increasing inexorable applications in industries and in present-day technology. The applications of the stretching sheet problem are such as polymer sheet extrusion from a dye, drawing, thinning and annealing of copper wires, glass fiber and paper production, and the cooling of a metallic plate in a cooling bath. The production of these sheets needs that the melt issues from a slit and is stretched to get the anticipated thickness. The final product depends on the rate of cooling in the process and the process of stretching. Sakadis [1] was the first to investigate the BL manners on a continuous solid surface moving with constant speed. Crane [2] was the first to attain a stylish analytical solution to the BL equations for the problem of steady $2 \mathrm{D}$ flow through a stretching surface in an inactive incompressible fluid. The work of Sakadis and Crane was extended and studied by several authors (Madaki et al. [3], Zheng et al. [4], Dessie and Kishan [5], and Pal [6]).

The rising need for chemical reaction and hydrometallurgical industries needs the study of heat and mass transfer with chemical reaction. There are several transport processes that are governed by the combined action of buoyancy forces due to both thermal and mass diffusion in the presence of chemical reactions and electric field effects. These processes occur in the nuclear reactor safety and combustion systems, solar collectors, metallurgical and chemical engineering, etc.

In real situations, most of the fluids used in engineering applications are almost non-Newtonian, specifically of the viscoelastic type than the viscous type. Khan [7], Madhu and Kishan [8], Babaelahi et al. [9], Raftari and Vajravelu [10], Goyal and Bhargava [11], and Madhu and Kishan [12] have investigated the flow of viscoelastic fluid flow under different conditions. Recently, Aliy and Kishan [13] examined the effect of electric field on MHD viscoelastic nanofluid flow and heat transfer over a stretching sheet with convective boundary conditions. They have considered the local nonsimilarity method and OHAM to solve the resulting equation. Jafar et al. [14] studied the MHD boundary layer flow of a viscoelastic fluid past a nonlinearly stretching sheet in the presence of a viscous dissipation effect.

Konda et al. [15] investigated the effect of nonuniform heat source/sink on MHD BLF and melting heat transfer of 
Williamson nanofluid in a porous medium. Hayat et al. [16] studied the effects of the chemical reaction of an unsteady 3D flow of the couple stress fluid over a stretching surface. Gireesha et al. [17] have studied the BLF and heat transfer of a dusty fluid flow over a stretching sheet in the existence of nonuniform heat source/sink and radiation. Parsa et al. [18] investigated the MHD boundary-layer flow over a stretching surface with internal heat generation or absorption. The thermal radiation effect was studied by different researchers (Weidman [19], Abel and Nandeppanavar [20], and Madaki et al. [21]).

OHAM is an approximate analytical technique that is directed forward to employ on a different type of problem, and the presence of any small or large parameters is not important. Marinca et al. [22] initially introduced the basic concept of this method in 2008. OHAM reduces the extent of the computational domain. It is a reliable analytical technique and has already been successfully applied to various nonlinear coupled differential equations occurring in science, engineering, and other fields of study. Marinca and Herisanu applied OHAM on the different problems (see, [23-25]). Many researchers applied OHAM to study fluid flow problems [26-29]. Recently, Gossaye and Kishan [13, 30, 31] applied the OHAM to the problem arisen from electrical MHD non-Newtonian fluid flows over a stretching sheet, and Khan et al. [32] used OHAM for Lane-Emden and Emden-Fowler initial and boundary value problems.

The novelty of the present study is starred due to the following aspects.

(i) Inclusion of electric field is justified since, for a remarkable enhancement in the value of the electric field, the struggle among fluid particles rises, and hence, the Lorentz force inclines to speed up the body forces, and it leads to escalating in the flow velocity and thicker momentum boundary layer. And also, we observed that the concentration of the fluid is affected by a large amount of an electric field.

(ii) The dimensionless ordinary differential equations with the corresponding boundary conditions are solved by using the approximate analytic technique OHAM.

The aim of this paper is to investigate the effect of electric field and thermal radiation on MHD viscoelastic fluid flow over a stretching surface through a porous medium with a heat source/sink. The strongly nonlinear differential equations are solved using the approximate analytic method OHAM. The effects of different governing parameters on velocity, temperature, and concentration profiles are displayed graphically and argued extensively. Moreover, the skin-friction coefficient is also exhibited using a table.

\section{Formulation of the Problem}

Consider a steady 2D boundary layer flow of an electrically conducting, viscoelastic fluid past a stretching surface embedded in a porous medium, the flow being confined to.
Two equal and opposite forces are applied along the axis, and hence, the surface is stretched, keeping the origin fixed. Assuming that a uniform magnetic field of strength is applied along that produces the magnetic effect in the direction.

The Lorentz force is given by $J \times B$, where $J=\sigma(E+V$ $\times B)$, the Joule current $J$ can be expressed as $J=\sigma(E+V \times$ $B), \sigma$ stands for the electrical conductivity, $V=(u, v)$ denotes the velocity of the fluid, and $B=\left(0, B_{0}, 0\right)$ and $E=\left(0,0,-E_{0}\right)$ are the transverse magnetic field and electric field vectors, correspondingly. The magnetic field and electric field are applied perpendicular to the flow, and hence, the Reynolds number is selected small. The induced magnetic field is insignificant for small magnetic Reynolds number because it is smaller when compared to the applied magnetic field. Under the usual $\mathrm{BL}$ conventions, the equations of continuity, momentum, energy, and concentration for the flow of viscoelastic fluid are:

$$
\begin{aligned}
& \frac{\partial v}{\partial y}+\frac{\partial u}{\partial x}=0 \\
& v \frac{\partial u}{\partial y}+u \frac{\partial u}{\partial x}=v \frac{\partial^{2} u}{\partial y^{2}}-\left(\frac{u \sigma B_{0}^{2}}{\rho}+\frac{u v}{K_{p}^{\prime}}\right)+\frac{k_{0}}{\rho}\left(\frac{\partial}{\partial x}\left(u \frac{\partial^{2} u}{\partial y^{2}}\right)\right. \\
& \left.+v \frac{\partial^{3} u}{\partial y^{3}}+\frac{\partial^{3} u}{\partial y^{3}} v+\frac{\partial u}{\partial y} \frac{\partial^{2} u}{\partial y \partial x}\right)+\frac{\sigma}{\rho} B_{0} E_{0}, \\
& \begin{aligned}
\left(u \frac{\partial T}{\partial x}+v \frac{\partial T}{\partial y}\right) \rho C_{p}- & k \frac{\partial^{2} T}{\partial y^{2}}-\mu\left(\frac{\partial u}{\partial y}\right)^{2} \\
= & k_{0}\left(\frac{\partial u}{\partial y} \frac{\partial}{\partial y}\left(u \frac{\partial u}{\partial x}+v \frac{\partial u}{\partial y}\right)\right) \\
& +\sigma B_{0}^{2} u^{2}-\frac{\partial q_{r}}{\partial y}+q\left(T-T_{\infty}\right),
\end{aligned} \\
& u \frac{\partial C}{\partial x}+v \frac{\partial C}{\partial y}+K_{c}^{\prime}\left(C-C_{\infty}\right)=D \frac{\partial^{2} C}{\partial y^{2}} .
\end{aligned}
$$

The approximation of Rosseland for thermal radiation gives $q_{r}=-(4 / 3)\left(\left(\sigma^{*} / 3 k_{1}\right)\left(\partial T^{4} / \partial y\right)\right)$. It is assumed that the temperature variation within the flow is such that may be expanded in a Taylor series. Expanding $T^{4}$ about $T_{\infty}$ and neglecting the higher-order terms, we have $T^{4}=4 T\left(T_{\infty}\right)^{3}$ $-3\left(T_{\infty}\right)^{4}$,

$$
\frac{\partial q_{r}}{\partial y}=-\frac{16}{3}\left(\frac{\sigma^{*} T_{\infty}^{3}}{\rho C_{p} k_{1}} \frac{\partial^{2} T}{\partial y^{2}}\right) .
$$

Taking the above equation and substituting it into equation (3), one can get 


$$
\begin{aligned}
u \frac{\partial T}{\partial x}+v \frac{\partial T}{\partial y}= & \alpha \frac{\partial^{2} T}{\partial y^{2}}+\frac{\mu}{\rho C_{p}}\left(\frac{\partial u}{\partial y}\right)^{2}+\left(\frac{16 \sigma^{*} T_{\infty}^{3}}{3 \rho C_{p} k_{1}}\right) \frac{\partial^{2} T}{\partial y^{2}} \\
& +\left(T-T_{\infty}\right) \frac{q}{\rho C_{p}}+\frac{\sigma B_{0}^{2}}{\rho C_{p}} u^{2}+\frac{k_{0}}{\rho C_{p}} \\
& \cdot\left(\frac{\partial u}{\partial y} \frac{\partial}{\partial y}\left(u \frac{\partial u}{\partial x}+v \frac{\partial u}{\partial y}\right)\right) .
\end{aligned}
$$

The boundary conditions are

$$
\left\{\begin{array}{l}
u=u_{w}=a x, v=v_{w}, T=T_{w}(x)=T_{\infty}+A\left(\frac{x}{L}\right)^{2} \text { (PST case), } \\
H_{w}(x)=-k\left(\frac{\partial T}{\partial y}\right)=B\left(\frac{x}{L}\right)^{2} \text { (PHF case), } \\
C=C_{w}(x)=C_{\infty}+a_{0}\left(\frac{x}{L}\right)^{2}, m_{w}=-D\left(\frac{\partial C}{\partial y}\right)=a_{1} x^{2} \text { as } y \rightarrow 0, \\
u=0, \frac{\partial u}{\partial y}=0, T \rightarrow T_{\infty}, C \rightarrow C_{\infty} \text { as } y \rightarrow \infty .
\end{array}\right.
$$

\section{Solution of the Flow Field}

Equations (1) and (2) disclose self-similar solutions of the type given below

$$
f=\frac{\psi}{v \sqrt{\mathrm{Re}_{x}}}, \eta=\frac{y}{x} \sqrt{\mathrm{Re}_{x}}
$$

where $f$ represents the dimensionless stream function, $\eta$ denotes the similarity variable, $\operatorname{Re}_{x}=u_{w} x / v$ represents the local Reynolds number, and $\psi(x, y)$ symbolizes the stream function satisfying the continuity equation (1). Substituting equation (8) in equation (2), we get

$$
\begin{gathered}
f^{\prime \prime \prime}+f f^{\prime \prime}-\left(f^{\prime}\right)^{2}+\operatorname{Rc}\left(2 f^{\prime} f^{\prime \prime \prime}-\left(f^{\prime \prime}\right)^{2}-f f^{i v}\right) \\
-\left(M+\frac{1}{K_{p}}\right) f^{\prime}+\mathrm{ME}_{1}=0,
\end{gathered}
$$

where $R c=k_{0} a / \mu$ is the viscoelastic parameter, $M=\sigma B_{0}^{2} / \rho a$ is the magnetic parameter, $E_{1}=E_{0} / u_{w} B_{0}$ is the electric field parameter, and $K_{p}=a K_{p}^{\prime} / v$ is the nondimensional permeability parameter.

The BCs are:

$$
f(0)=f_{w}, f^{\prime}(0)=1, f^{\prime}(\infty)=0, f^{\prime \prime}(\infty)=0,
$$

where $f_{w}=-v_{w} \sqrt{\operatorname{Re}_{x}} / u_{w}$ is the suction/injection parameter, and $f_{w}>0$ and $f_{w}<0$ represent suction and injection, respectively.
Now, we apply OHAM to the nonlinear ODE (9) with the BCs (10) under the following assumption

$$
\begin{aligned}
f & =f_{0}+p f_{1}+p^{2} f_{2}, g=g_{0}+p g_{1}+p^{2} g_{2}, \\
G & =G_{0}+p G_{1}+p^{2} G_{2}, h=h_{0}+p h_{1}+p^{2} h_{2}, \\
H_{1}(p) & =C_{1} p+C_{2} p^{2}, H_{2}(p)=C_{3} p+C_{4} p^{2}, \\
H_{3}(p) & =C_{5} p+C_{6} p^{2}, H_{4}(p)=C_{7} p+C_{8} p^{2},
\end{aligned}
$$

where the parameter $p \in[0,1], H_{i}(p), i=1,2,3,4$ is an auxiliary function which is different from zero, and $C_{j},(j=1,2,3,4,5,6)$ are constants.

And also, assume the following

$$
\begin{aligned}
L= & f^{\prime}+f^{\prime \prime}, \\
N= & f^{\prime \prime \prime}+f f^{\prime \prime}-\left(f^{\prime}\right)^{2}+\operatorname{Rc}\left(2 f^{\prime} f^{\prime \prime \prime}-\left(f^{\prime \prime}\right)^{2}-f f^{i v}\right) \\
& -\left(M+\frac{1}{K_{p}}\right) f^{\prime}+M E_{1}-f^{\prime}-f^{\prime \prime},
\end{aligned}
$$

where $N$ and $L$ are the nonlinear and linear operators, respectively. Hence, the OHAM family of equations is given by

$$
\begin{aligned}
& H_{1}(p)\left(-\left(f^{\prime}\right)^{2}+\operatorname{Rc}\left(2 f^{\prime} f^{\prime \prime \prime}-\left(f^{\prime \prime}\right)^{2}-f f^{i v}\right)\right. \\
& \left.-\left(M+\frac{1}{K_{p}}\right) f^{\prime}+M E_{1}+f^{\prime \prime \prime}+f f^{\prime \prime}\right) \\
& -\left(f^{\prime}+f^{\prime \prime}\right)(1-p)=0 .
\end{aligned}
$$

Using the BCs (10) and comparing the coefficients of the same powers of $p$-terms, we can get the hypothesis below:

Equating the coefficients of the zero-order equation $p^{0}$, we obtain

$f_{0}{ }^{\prime \prime}+f_{0}{ }^{\prime}=0, f_{0}(0)=f_{w}, f_{0}{ }^{\prime}(0)=1, f_{0}{ }^{\prime}(\infty)=0, f_{0}{ }^{\prime \prime}(\infty)=0$.

Equating the coefficients of the first-order equation $p^{1}$, we obtain

$$
\begin{aligned}
& {\left[-\left(f_{0}{ }^{\prime}\right)^{2}+\operatorname{Rc}\left(2 f_{0}{ }^{\prime} f_{0}{ }^{\prime \prime \prime}-\left(f_{0}{ }^{\prime \prime}\right)^{2}-f_{0} f_{0}{ }^{i v}\right)\right.} \\
& \left.\quad-\left(M+\frac{1}{K_{p}}\right) f_{0}{ }^{\prime}+M E_{1}+f_{0}{ }^{\prime \prime \prime}+f_{0} f_{0}{ }^{\prime \prime}\right] C_{1}-f_{1}{ }^{\prime}-f_{1}{ }^{\prime \prime} \\
& \quad+f_{0}{ }^{\prime \prime}+f_{0}{ }^{\prime}=0,
\end{aligned}
$$

$$
f_{1}(0)=0, f_{1}^{\prime}(0)=0, f_{1}^{\prime}(\infty)=0, f_{1}^{\prime \prime}(\infty)=0
$$


Equating the second-order equation $p^{2}$, we obtain

$$
\begin{aligned}
f_{2}{ }^{\prime \prime}+f_{2}{ }^{\prime}= & C_{1}\left[\operatorname{Rc}\left(2 f_{1}{ }^{\prime} f_{0}{ }^{\prime \prime \prime}+2 f_{0}{ }^{\prime} f_{1}{ }^{\prime \prime \prime}-2 f_{0}{ }^{\prime \prime} f_{1}{ }^{\prime \prime}-f_{1} f_{0}{ }^{i v}-f_{0} f_{1}{ }^{i v}\right)\right. \\
& -\left(M+\frac{1}{K_{p}}\right) f_{1}{ }^{\prime}+M E_{1}+f_{1}{ }^{\prime \prime \prime}+f_{1} f_{0}{ }^{\prime \prime}+f_{0} f_{1}{ }^{\prime \prime} \\
& \left.-2 f_{0} f_{1}^{\prime}\right]+f_{1}{ }^{\prime}+f_{1}{ }^{\prime \prime}+C_{2} \\
& \cdot\left[-\left(f_{0}{ }^{\prime}\right)^{2}+\operatorname{Rc}\left(2 f_{0}{ }^{\prime} f_{0}{ }^{\prime \prime}-\left(f_{0}{ }^{\prime \prime}\right)^{2}-f_{0} f_{0}{ }^{i v}\right)\right. \\
& \left.-\left(M+\frac{1}{K_{p}}\right) f_{0}{ }^{\prime}+M E_{1}+f_{0}{ }^{\prime \prime \prime}+f_{0} f_{0}{ }^{\prime \prime}\right], \\
f_{2}(0)= & 0, f_{2}{ }^{\prime}(0)=0, f_{2}{ }^{\prime}(\infty)=0, f_{2}{ }^{\prime \prime}(\infty)=0 .
\end{aligned}
$$

After solving the ODEs (14) and (15) with the corresponding boundary conditions, we obtain

$$
\begin{aligned}
f_{0}= & e^{-\eta}\left(-1+e^{\eta}+e^{\eta} f_{w}\right), \\
f_{1}= & -\frac{1}{K_{p}} c 1 e^{-2 \eta}\left(-e^{\eta}+e^{2 \eta}-e^{\eta} f_{w} K_{p}+e^{2 \eta} f_{w} K_{p}-e^{\eta} K_{p} M\right. \\
& +e^{2 \eta} K_{p} M-e^{\eta} E_{1} K_{p} M+e^{2 \eta} E_{1} K_{p} M-K_{p} \mathrm{Rc}+e^{\eta} K_{p} \mathrm{Rc} \\
& -e^{\eta} f_{w} K_{p} \mathrm{Rc}+e^{2 \eta} f_{w} K_{p} \mathrm{Rc}-e^{\eta} \eta-e^{\eta} f_{w} K_{p} \eta-e^{\eta} K_{p} M \eta \\
& \left.-e^{2 \eta} E_{1} K_{p} M \eta-e^{\eta} K_{p} R c \eta-e^{\eta} f_{w} K_{p} R c \eta\right) .
\end{aligned}
$$

We cannot write the third term $f_{2}$ here because of its being large. Hence, the solution $f\left(\eta, C_{i}\right)$ in terms of the parameters can be written as:

$$
f\left(\eta, C_{i}\right)=f_{0}(\eta)+f_{1}\left(\eta, C_{i}\right)+f_{2}\left(\eta, C_{i}\right), i=1,2 .
$$

For equation (19), we define its residual equation in the form given below

$$
\begin{aligned}
R_{1}\left(\eta, C_{i}\right)= & f^{\prime \prime \prime}+f f^{\prime \prime}-\left(f^{\prime}\right)^{2}+\operatorname{Rc}\left(2 f^{\prime} f^{\prime \prime \prime}-\left(f^{\prime \prime}\right)^{2}-f f^{i v}\right) \\
& -\left(M+\frac{1}{K_{p}}\right) f^{\prime}+M E_{1} .
\end{aligned}
$$

The unknown convergence parameters $C_{1}$ and $C_{2}$ will be optimally well-known using the conditions given below

$$
\frac{\partial J_{1}\left(C_{i}\right)}{\partial C_{1}}=\frac{\partial J_{1}\left(C_{i}\right)}{\partial C_{2}}=0, \text { where } J_{1}\left(C_{i}\right)=\int_{0}^{0.1} R_{1}^{2}\left(\eta, C_{i}\right) d \eta .
$$

Taking particular cases when $K_{p}=100, R c=0.5, M=2$, $E_{1}=0.1$, and $f_{w}=1$, the convergence parameters are as follows

$$
C_{1}=0.14227123495837554, C_{2}=-0.0985828777094099 .
$$

Hence, the approximate analytical solution is given as

$$
\begin{aligned}
f(\eta)= & e^{-\eta}\left(-1+2 e^{\eta}\right)-0.28454246991511 e^{-2 \eta} \\
& \cdot\left(-0.25-2.6 e^{\eta}+2.85 e^{2 \eta}-3 . e^{\eta} \eta+0.1 e^{2 \eta} \eta\right) \\
& +0.666666666666 e^{-3 \eta}(0.0012650690185363315 \\
& -0.2435248057116105 e^{\eta}-0.6482617721497719 e^{2 \eta} \\
& +0.8905215088428458 e^{3 \eta}-0.09108496933461585 e^{\eta} \eta \\
& -1.0292483892015603 e^{2 \eta} \eta-0.01118281798120787 e^{3 \eta} \eta \\
& \left.-0.3597856288717327 e^{2 \eta} \eta^{2}\right) .
\end{aligned}
$$

The shear stress at the wall is defined as

$$
\tau_{w}=\mu\left(\frac{\partial u}{\partial y}\right)_{y=0}=\mu a x \sqrt{\frac{a}{v f^{\prime \prime}(0)}} .
$$

The nondimensional form of skin friction coefficient at the well is

$$
f^{\prime \prime}(0)=-C_{f} \operatorname{Re}_{x}^{1 / 2}
$$

\section{Heat Transfer Analysis}

4.1. Case I: Prescribed Surface Temperature (PST). In the prescribed surface temperature case, introducing nondimensional quantities as given below

$$
\begin{aligned}
g(\eta) & =\frac{T-T_{\infty}}{T_{w}-T_{\infty}}, \operatorname{Pr}=\frac{v}{\alpha}, \mathrm{Ec}=\frac{a^{2} L^{2}}{A C_{p}}, \operatorname{Rc}=\frac{a k_{0}}{\mu}, Q \\
& =\frac{q}{a \rho C_{p}}, \operatorname{Rd}=\frac{16 \sigma^{*} T_{\infty}^{3}}{3 k k_{1}},
\end{aligned}
$$

and using equation (7) equation (6) become

$$
\begin{aligned}
(\mathrm{Rd}+1) g^{\prime \prime}+ & \operatorname{Pr}\left(f g^{\prime}+\left(Q-2 f^{\prime}\right) g+\mathrm{Ec}\left(\left(f^{\prime \prime}\right)^{2}\right.\right. \\
& \left.\left.+\operatorname{Rc} f^{\prime \prime}\left(f^{\prime} f^{\prime \prime}-f f^{\prime \prime \prime}\right)+\left(M+\frac{1}{K_{p}}\right)\left(f^{\prime}\right)^{2}\right)\right)=0,
\end{aligned}
$$

with the BCs given below

$$
g(0)=1, g(\infty)=0 .
$$

Now let us apply the OHAM to the nonlinear ODE (27) using the following assumption 


$$
\begin{aligned}
L= & g^{\prime}+g, \quad N=(\mathrm{Rd}+1) g^{\prime \prime}+\operatorname{Pr} \\
& \cdot\left(f g^{\prime}+\left(Q-2 f^{\prime}\right) g+\operatorname{Ec}\left(\left(f^{\prime}\right)^{2}+\operatorname{Rc} f^{\prime \prime}\left(f^{\prime} f^{\prime \prime}-f f^{\prime \prime \prime}\right)\right.\right. \\
& \left.\left.+\left(M+\frac{1}{K_{p}}\right)\left(f^{\prime}\right)^{2}\right)\right)-\left(g^{\prime}+g\right),
\end{aligned}
$$

where $N$ and $L$ are the nonlinear and linear operators, respectively. Hence, the OHAM family of equations is given by

$$
\begin{aligned}
\left(g^{\prime}+g\right)(1-p)= & H_{2}(p)\left((\mathrm{Rd}+1) g^{\prime \prime}+\operatorname{Pr}\left(f g^{\prime}+\left(Q-2 f^{\prime}\right) g\right.\right. \\
& +\operatorname{Ec}\left(\left(f^{\prime}\right)^{2}+\operatorname{Rc} f^{\prime \prime}\left(f^{\prime} f^{\prime \prime}-f f^{\prime \prime \prime}\right)\right. \\
& \left.\left.\left.+\left(M+\frac{1}{K_{p}}\right)\left(f^{\prime}\right)^{2}\right)\right)\right) .
\end{aligned}
$$

Using the BCs (7) and comparing the coefficients of the same powers of $p$-terms in the above equation, one can have the following:

Equating the coefficients of the zero-order equation $p^{0}$, we obtain

$$
g_{0}{ }^{\prime}+g_{0}=0, g_{0}(0)=1, g_{0}(\infty)=0 .
$$

Equating the coefficients of the first-order equation $p^{1}$, we get

$$
\begin{aligned}
g_{1}{ }^{\prime}+g_{1}= & g_{0}{ }^{\prime}+g_{0}+C_{3}\left((\operatorname{Rd}+1) g_{0}{ }^{\prime \prime}+\operatorname{Pr}\left(f_{0} g_{0}{ }^{\prime}+\left(Q-2 f_{0}{ }^{\prime}\right) g_{0}\right.\right. \\
& +\mathrm{Ec}\left(\operatorname{Rc} f_{0}{ }^{\prime \prime}\left(f_{0}{ }^{\prime} f_{0}{ }^{\prime \prime}-f_{0} f_{0}{ }^{\prime \prime}\right)+\left(M+\frac{1}{K_{p}}\right)\left(f_{0}{ }^{\prime}\right)^{2}\right. \\
& \left.\left.\left.+\left(f_{0}{ }^{\prime}\right)^{2}\right)\right)\right), g_{1}(0)=0, g_{1}(\infty)=0 .
\end{aligned}
$$

Equating the coefficients of the second-order equation $p^{2}$, we get

$$
\begin{aligned}
g_{2}{ }^{\prime}+g_{2}= & g_{1}{ }^{\prime}+g_{1}+C_{3} \\
& \cdot\left((\operatorname{Rd}+1) g_{1}{ }^{\prime \prime}+\operatorname{Pr}\left(f_{1} g_{0}{ }^{\prime}+f_{0} g_{1}{ }^{\prime}+\left(Q-2 f_{1}{ }^{\prime}\right) g_{1}\right.\right. \\
& \left.\left.+\mathrm{Ec}\left(\begin{array}{c}
\operatorname{Rc} f_{0}{ }^{\prime \prime}\left(f_{1}{ }^{\prime} f_{0}{ }^{\prime \prime}+f_{0}{ }^{\prime} f_{1}{ }^{\prime}-f_{1} f_{0}{ }^{\prime \prime}-f_{0} f_{1}{ }^{\prime \prime \prime}\right)+2 f_{1}{ }^{\prime \prime} f_{0}{ }^{\prime \prime} \\
2\left(M+\frac{1}{K_{p}}\right) f_{1}{ }^{\prime} f_{0}{ }^{\prime}+\operatorname{Rc} f_{1}{ }^{\prime \prime}\left(f_{0}{ }^{\prime} f_{0}{ }^{\prime \prime}-f_{0} f_{0}{ }^{\prime \prime \prime}\right)
\end{array}\right)\right)\right) \\
& +C_{4}\left((\operatorname{Rd}+1) g_{0}{ }^{\prime \prime}+\operatorname{Pr}\left(f_{0} g_{0}{ }^{\prime}+\left(Q-2 f_{0}{ }^{\prime}\right) g_{0}\right.\right. \\
& +\mathrm{Ec}\left(\operatorname{Rc} f_{0}{ }^{\prime \prime}\left(f_{0}{ }^{\prime} f_{0}{ }^{\prime \prime}-f_{0} f_{0}{ }^{\prime \prime}\right)+\left(M+\frac{1}{K_{p}}\right)\left(f_{0}{ }^{\prime}\right)^{2}\right. \\
& \left.\left.\left.+\left(f_{0}{ }^{\prime}\right)^{2}\right)\right)\right),
\end{aligned}
$$

$$
g_{2}(0)=0 \text {.and } g_{2}(\infty)=0 \text {. }
$$

After solving the ODEs (31), (34), and (36) with the corresponding boundary conditions, we obtain

$$
\begin{aligned}
g_{0}= & e^{-\eta} \\
g_{1}= & \frac{1}{2 K_{p}} C_{3} e^{-3 \eta}\left(-2 e^{\eta} \mathrm{EcPr}+2 e^{2 \eta} \mathrm{EcPr}+2 e^{\eta} K_{p} \operatorname{Pr}-2 e^{2 \eta} K_{p} \operatorname{Pr}\right. \\
& -2 e^{\eta} \mathrm{Ec} K_{p} \operatorname{Pr}+2 e^{2 \eta} \mathrm{Ec} K_{p} \operatorname{Pr}-2 e^{\eta} \mathrm{Ec} K_{p} M \operatorname{Pr} \\
& +2 e^{2 \eta} \mathrm{E} K_{p} M \operatorname{Pr}+\mathrm{Ec} K_{p} \operatorname{PrRc}-2 e^{\eta} \mathrm{Ec} f_{w} K_{p} \operatorname{PrRc} \\
& +2 e^{2 \eta} \mathrm{Ec} f_{w} K_{p} \operatorname{PrRc}+2 e^{2 \eta} K_{p} \eta-2 e^{2 \eta} K_{p} \operatorname{Pr} \eta \\
& -2 e^{2 \eta} f_{w} K_{p} \operatorname{Pr} \eta+2 e^{2 \eta} K_{p} \operatorname{Pr} Q \eta+2 e^{2 \eta} K_{p} \operatorname{Rd} \eta \\
& \left.-e^{2 \eta} \mathrm{Ec} K_{p} \operatorname{PrRc}\right) .
\end{aligned}
$$

We cannot write the third-term $g_{2}$ here because of it being large. Hence, the solution $g\left(\eta, C_{i}\right)$ in terms of the parameters can be written as:

$$
g\left(\eta, C_{i}\right)=g_{0}(\eta)+g_{1}\left(\eta, C_{i}\right)+g_{2}\left(\eta, C_{i}\right), i=1,2,3,4 .
$$

For equation (36), we define its residual equation in the form

$$
\begin{aligned}
R_{2}\left(\eta, C_{i}\right)= & (\mathrm{Rd}+1) g^{\prime \prime}+\operatorname{Pr}\left(f g^{\prime}+\left(Q-2 f^{\prime}\right) g\right. \\
& +\operatorname{Ec}\left(\operatorname{Rc} f^{\prime \prime}\left(f^{\prime} f^{\prime \prime}-f f^{\prime \prime \prime}\right)+\left(M+\frac{1}{K_{p}}\right)\right. \\
& \left.\left.\cdot\left(f^{\prime}\right)^{2}+\left(f^{\prime \prime}\right)^{2}\right)\right) .
\end{aligned}
$$

The unknown convergence parameters $C_{3}$ and $C_{4}$ can be optimally obtained from the following expressed conditions given below

$$
\begin{aligned}
\frac{\partial J_{2}\left(C_{i}\right)}{\partial C_{1}} & =\frac{\partial J_{2}\left(C_{i}\right)}{\partial C_{2}}=\frac{\partial J_{2}\left(C_{i}\right)}{\partial C_{3}}=\frac{\partial J_{2}\left(C_{i}\right)}{\partial C_{4}}=0, \text { where } J_{2}\left(C_{i}\right) \\
& =\int_{0}^{0.1} R_{2}^{2}\left(\eta, C_{i}\right) d \eta .
\end{aligned}
$$

In the particular case when $\mathrm{Rc}=K_{p}=0.5, E_{1}=\mathrm{Ec}=0.1$, $f_{w}=1, M=\operatorname{Pr}=2, Q=\operatorname{Rd}=0.2$, one can obtain the results of the convergence parameters. Hence, the values are calculated and obtained below

$$
\begin{array}{ll}
C_{1}=0.14227123495837554, & C_{2}=-0.0985828777094099, \\
C_{3}=0.33085325050763764, & C_{4}=-0.17615393227116716 .
\end{array}
$$


After substituting all the parameters, we get

$$
\begin{aligned}
g(\eta)= & e^{-\eta}+e^{-3 \eta}\left(0.016542662525381883+0.2977679254568738 e^{\eta}\right. \\
& \left.-0.31431058798225564 e^{2 \eta}-0.7940478012183303 e^{2 \eta} \eta\right) \\
& +0.3333333333333333 e^{-4 \eta} \\
& \cdot\left(-0.018007022418104122+0.09855963046417679 e^{\eta}\right. \\
& -1.262581048766744 e^{2 \eta}+1.1820284407206736 e^{3 \eta} \\
& -1.2401935466956018 e^{2 \eta} \eta-0.08408331700268 e^{3 \eta} \eta \\
& \left.+0.9175253256056694 e^{3 \eta} \eta^{2}\right) .
\end{aligned}
$$

The local Nusselt number for PST case is given by

$$
N u_{x} \operatorname{Re}_{x}^{-1 / 2}=-g^{\prime}(0)
$$

4.2. Case II: Prescribed Heat Flux (PHF). In the prescribed heat flux case, introducing the similarity variable $T=T_{\infty}+$ $G(\eta)\left(B x^{2} / k L^{2}\right) \sqrt{v / a}$ and using equation (7), equation (6) becomes

$$
\begin{aligned}
(\mathrm{Rd}+1) G^{\prime \prime}+ & \operatorname{Pr}\left(f G^{\prime}+\left(Q-2 f^{\prime}\right) G+\operatorname{Ec}\left(\left(f^{\prime}\right)^{2}+\operatorname{Rc} f^{\prime \prime}\right.\right. \\
& \left.\left.\cdot\left(f^{\prime} f^{\prime \prime}-f f^{\prime \prime \prime}\right)+\left(M+\frac{1}{K_{p}}\right)\left(f^{\prime}\right)^{2}\right)\right)=0,
\end{aligned}
$$

with the boundary conditions

$$
G^{\prime}(0)=-1, G(\infty)=0
$$

Now, let us apply the OHAM to the nonlinear ODE (42) using the following assumption

$$
\begin{aligned}
L= & G+G^{\prime}, \quad N=(\mathrm{Rd}+1) G^{\prime \prime}+\operatorname{Pr}\left(f G^{\prime}+\left(Q-2 f^{\prime}\right) G+\mathrm{Ec}\right. \\
& \cdot\left(\left(f^{\prime}\right)^{2}+\mathrm{Rc} f^{\prime \prime}\left(f^{\prime} f^{\prime \prime}-f f^{\prime \prime \prime}\right)\right. \\
& \left.\left.+\left(M+\frac{1}{K_{p}}\right)\left(f^{\prime}\right)^{2}\right)\right)-\left(G^{\prime}+G\right),
\end{aligned}
$$

where $N$ and $L$ are the nonlinear and linear operators, respectively. Hence, the OHAM family of equations is given by

$$
\begin{aligned}
\left(G^{\prime}+G\right)(1-p)= & H_{3}(p)\left((\operatorname{Rd}+1) G^{\prime \prime}+\operatorname{Pr}\left(f G^{\prime}+\left(Q-2 f^{\prime}\right) G\right.\right. \\
& +\operatorname{Ec}\left(\left(f^{\prime}\right)^{2}+\operatorname{Rc} f^{\prime \prime}\left(f^{\prime} f^{\prime \prime}-f f^{\prime \prime \prime}\right)\right. \\
& \left.\left.\left.+\left(M+\frac{1}{K_{p}}\right)\left(f^{\prime}\right)^{2}\right)\right)\right) .
\end{aligned}
$$

Using the BCs (7) and comparing the coefficients of the same powers of $p$-terms in the above equation, one can have the following:
Equating the coefficients of the zero-order equation $p^{0}$, we obtain

$$
G_{0}{ }^{\prime}+G_{0}=0, G_{0}{ }^{\prime}(0)=-1, G_{0}(\infty)=0 .
$$

Equating the coefficients of the first-order equation $p^{1}$, we get

$$
\begin{aligned}
G_{1}{ }^{\prime}+G_{1}= & G_{0}{ }^{\prime}+G_{0}+C_{5}\left((\operatorname{Rd}+1) G_{0}{ }^{\prime \prime}+\operatorname{Pr}\left(f_{0} G_{0}{ }^{\prime}+\left(Q-2 f_{0}{ }^{\prime}\right) G_{0}\right.\right. \\
& +\operatorname{Ec}\left(\operatorname{Rc} f_{0}{ }^{\prime \prime}\left(f_{0}^{\prime} f_{0}{ }^{\prime \prime}-f_{0} f_{0}{ }^{\prime \prime \prime}\right)+\left(M+\frac{1}{K_{p}}\right)\left(f_{0}{ }^{\prime}\right)^{2}\right. \\
& \left.\left.\left.+\left({f_{0}^{\prime}}^{\prime}\right)^{2}\right)\right)\right), G_{1}{ }^{\prime}(0)=0, G_{1}(\infty)=0 .
\end{aligned}
$$

Equating the coefficients of the second-order equation $p^{2}$, we get

$$
\begin{aligned}
G_{2}{ }^{\prime}+G_{2}= & G_{1}{ }^{\prime}+G_{1}+C_{5} \\
& \cdot\left((\operatorname{Rd}+1) G_{1}{ }^{\prime \prime}+\operatorname{Pr}\left(f_{1} G_{0}{ }^{\prime}+f_{0} G_{1}{ }^{\prime}+\left(Q-2 f_{1}{ }^{\prime}\right) G_{1}\right.\right. \\
& \left.+\operatorname{Ec}\left(\begin{array}{c}
\left.\operatorname{Rc} f_{0}{ }^{\prime \prime}\left(f_{1}{ }^{\prime} f_{0}{ }^{\prime}+f_{0}{ }^{\prime} f_{1}{ }^{\prime \prime}-f_{1} f_{0}{ }^{\prime \prime \prime}-f_{0} f_{1}{ }^{\prime \prime \prime}\right)+2 f_{1}{ }^{\prime \prime} f_{0}{ }^{\prime \prime}\right) \\
2\left(M+\frac{1}{K_{p}}\right) f_{1}{ }^{\prime} f_{0}{ }^{\prime}+\operatorname{Rc} f_{1}{ }^{\prime \prime}\left(f_{0}{ }^{\prime} f_{0}{ }^{\prime \prime}-f_{0} f_{0}{ }^{\prime \prime \prime}\right)
\end{array}\right)\right) \\
& +C_{6}\left((\operatorname{Rd}+1) G_{0}{ }^{\prime \prime}+\operatorname{Pr}\left(f_{0} G_{0}{ }^{\prime}+\left(Q-2 f_{0}{ }^{\prime}\right) G_{0}\right.\right. \\
& \left.\left.+\operatorname{Ec}\left(\operatorname{Rc} f_{0}{ }^{\prime \prime}\left(f_{0}{ }^{\prime} f_{0}{ }^{\prime \prime}-f_{0} f_{0}{ }^{\prime \prime \prime}\right)+\left(M+\frac{1}{K_{p}}\right)\left(f_{0}{ }^{\prime}\right)^{2}+\left(f_{0}{ }^{\prime}\right)^{2}\right)\right)\right),
\end{aligned}
$$

$G_{2}^{\prime}(0)=0$ and $G_{2}(\infty)=0$.

After solving the ODEs (46), (47), and (48) with the corresponding boundary conditions, we obtain

$$
\begin{aligned}
G_{0}=e^{-\eta} & \\
G_{1}= & \frac{1}{2 K_{p}} C_{5} e^{-3 \eta}\left(2 e^{2 \eta} K_{p}-2 e^{\eta} \mathrm{EcPr}+4 e^{2 \eta} \mathrm{EcPr}+2 e^{\eta} K_{p} \operatorname{Pr}\right. \\
& -6 e^{2 \eta} K_{p} \operatorname{Pr}-2 e^{\eta} \mathrm{Ec} K_{p} \operatorname{Pr}+4 e^{2 \eta} \mathrm{Ec} K_{p} \operatorname{Pr} \\
& -2 e^{2 \eta} f_{w} K_{p} \operatorname{Pr}-2 e^{\eta} \mathrm{Ec} K_{p} M \operatorname{Pr}+4 e^{2 \eta} \mathrm{Ec} K_{p} M \operatorname{Pr} \\
& +\mathrm{E} c K_{p} \operatorname{Pr} \operatorname{Rc}-3 e^{2 \eta} \mathrm{E} c K_{p} \operatorname{Pr} \operatorname{Rc}-2 e^{\eta} \mathrm{E} c f_{w} K_{p} \operatorname{PrRc} \\
& +4 e^{2 \eta} \operatorname{Ec} f_{w} K_{p} \operatorname{Pr} \operatorname{Rc}+2 e^{2 \eta} K_{p} \operatorname{Rd}+2 e^{2 \eta} K_{p} \eta \\
& -2 e^{2 \eta} K_{p} \operatorname{Pr} \eta-2 e^{2 \eta} f_{w} K_{p} \operatorname{Pr} \eta+2 e^{2 \eta} K_{p} \operatorname{Pr} Q \eta \\
& \left.+2 e^{2 \eta} K_{p} \operatorname{Rd} \eta\right)+2 e^{2 \eta} K_{p} \operatorname{Pr} Q .
\end{aligned}
$$

We cannot write the third term $G_{2}$ here because of it being large. Hence, the solution $G\left(\eta, C_{i}\right)$ in terms of the parameters can be written as:

$$
G\left(\eta, C_{i}\right)=G_{0}(\eta)+G_{1}\left(\eta, C_{i}\right)+G_{2}\left(\eta, C_{i}\right), i=1,2,5,6 .
$$
form

For equation (50), we define its residual equation in the 


$$
\begin{aligned}
R_{3}\left(\eta, C_{i}\right)= & (\operatorname{Rd}+1) G^{\prime \prime}+\operatorname{Pr}\left(f G^{\prime}+\left(Q-2 f^{\prime}\right) G\right. \\
& +\mathrm{Ec}\left(\operatorname{Rc} f^{\prime \prime}\left(f^{\prime} f^{\prime \prime}-f f^{\prime \prime \prime}\right)+\left(M+\frac{1}{K_{p}}\right)\right. \\
& \left.\left.\cdot\left(f^{\prime}\right)^{2}+\left(f^{\prime}\right)^{2}\right)\right) .
\end{aligned}
$$

The unknown convergence parameters $C_{3}$ and $C_{4}$ can be optimally obtained from the following expressed conditions given below

$$
\begin{aligned}
\frac{\partial J_{3}\left(C_{i}\right)}{\partial C_{1}} & =\frac{\partial J_{3}\left(C_{i}\right)}{\partial C_{2}}=\frac{\partial J_{3}\left(C_{i}\right)}{\partial C_{5}}=\frac{\partial J_{3}\left(C_{i}\right)}{\partial C_{6}} \\
& =0 \text {, where } J_{3}\left(C_{i}\right)=\int_{0}^{0.1} R_{3}^{2}\left(\eta, C_{i}\right) d \eta .
\end{aligned}
$$

In a particular case when $\mathrm{Rc}=K_{p}=0.5, E_{1}=\mathrm{Ec}=0.1$, $f_{w}=1, M=\operatorname{Pr}=2, Q=\operatorname{Rd}=0.2$, one can obtain the results of the convergence parameters. Hence, the values are calculated and obtained below

$$
\begin{aligned}
& C_{1}=0.14227123495837554, \quad C_{2}=-0.0985828777094099, \\
& C_{5}=0.10515520472075117, \quad C_{6}=0.006461970319935396 .
\end{aligned}
$$

After substituting all the parameters, we get

$$
\begin{aligned}
G(\eta)= & e^{-\eta}+e^{-3 \eta}(0.005257760236037559 \\
& +0.09463968424867605 e^{\eta}-0.45742514053526756 e^{2 \eta} \\
& \left.-0.2523724913298028 e^{2 \eta} \eta\right)+0.3333333333333333 e^{-4 \eta} \\
& \cdot\left(-0.0033498458336746195+0.029901242487034338 e^{\eta}\right. \\
& +0.16239147539668197 e^{2 \eta}-0.5480934455483598 e^{3 \eta} \\
& -0.05241128156726507 e^{2 \eta} \eta-0.09459486906132786 e^{3 \eta} \eta \\
& \left.+0.08656147506726386 e^{3 \eta} \eta^{2}\right) .
\end{aligned}
$$

The local Nusselt number for the PHF case is given by

$$
N u_{x} \operatorname{Re}_{x}^{-1 / 2}=\frac{1}{G(0)}
$$

\section{Mass Transfer Analysis}

Introducing the similarity variables $C-C_{\infty}=h(\eta)\left(a_{1} x^{2} / D\right)$ $\sqrt{v / a}$ and using equation (7) in equation (4), we obtain the following

$$
h^{\prime \prime}+\mathrm{Sc}\left(f h^{\prime}-f^{\prime} h-\mathrm{Kch}\right)=0 \text {, }
$$

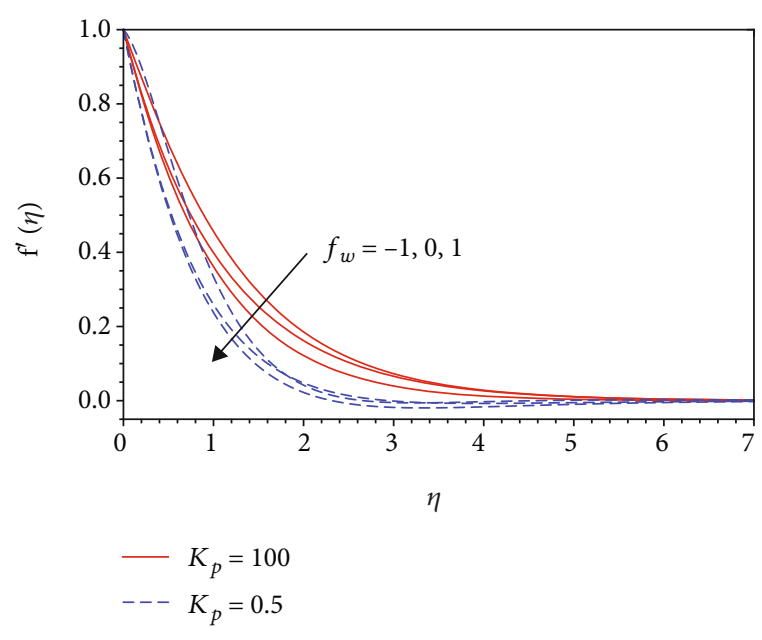

FIgURE 1: Velocity profiles (second-grade fluid) for different values of $f_{w}$ when $M=0.5$ and $\mathrm{Rc}=1$.

with boundary condition

$$
h^{\prime}(0)=-1, \quad h(\infty)=0 .
$$

Now, let us apply the OHAM to the nonlinear ODE (56) using the following assumption

$$
L=h^{\prime}+h, N=h^{\prime \prime}+\mathrm{Sc}\left(f h^{\prime}-f^{\prime} h-\mathrm{Kc} h\right)-\left(h+h^{\prime}\right),
$$

where $L$ and $N$ stands for the linear and nonlinear operators. Consequently, the OHAM family of equations is written as

$$
\left(h^{\prime}+h\right)(1-p)=H_{4}(p)\left(h^{\prime \prime}+\operatorname{Sc}\left(f h^{\prime}-f^{\prime} h-\mathrm{Kch}\right)\right) .
$$

Using the BCs (7) and comparing the coefficients of the same powers of $p$-terms, one can have the following:

Equating the coefficients of the zero-order equation $p^{0}$, one can obtain

$$
h_{0}{ }^{\prime}+h_{0}=0, h_{0}{ }^{\prime}(0)=-1, h_{0}(\infty)=0 \text {. }
$$

Equating the coefficients of the first-order equation $p^{1}$, we get

$$
h_{1}{ }^{\prime}+h_{1}=h_{0}{ }^{\prime}+h_{0}+C_{7}\left(h_{0}{ }^{\prime}+\operatorname{Sc}\left(f_{0} h_{0}{ }^{\prime}-f_{0}{ }^{\prime} h_{0}-\mathrm{Kch} h_{0}\right)\right) \text {, }
$$

$$
h_{1}^{\prime}(0)=0, \quad h_{1}(\infty)=0 .
$$

Equating the coefficients of the second-order equation $p^{2}$, we get 


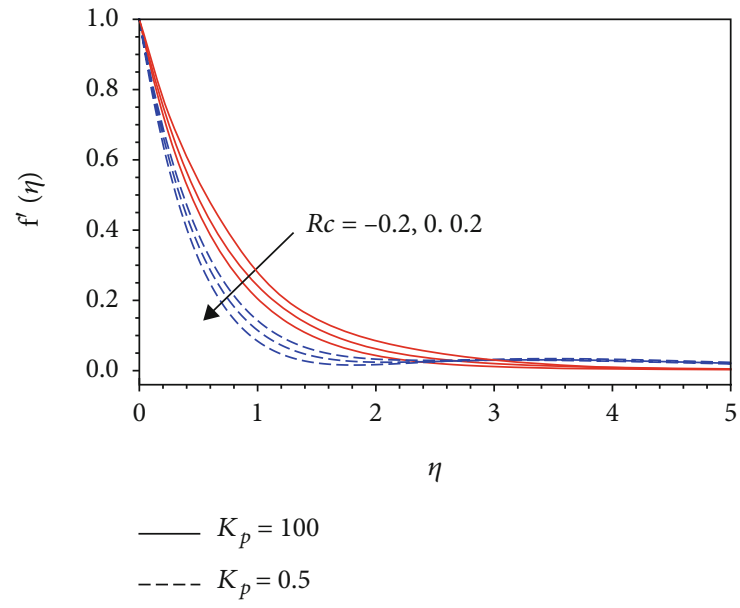

Figure 2: Velocity profiles for dissimilar values of Rc when $M=1$ and $f_{w}=0$.

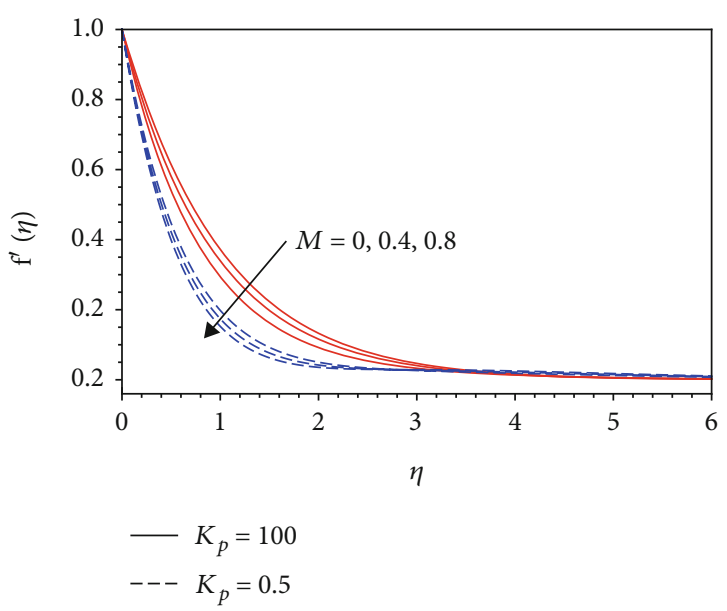

Figure 3: Velocity profile for dissimilar values of $M$ when $\mathrm{Rc}=0.2, f_{w}=E_{1}=0$.

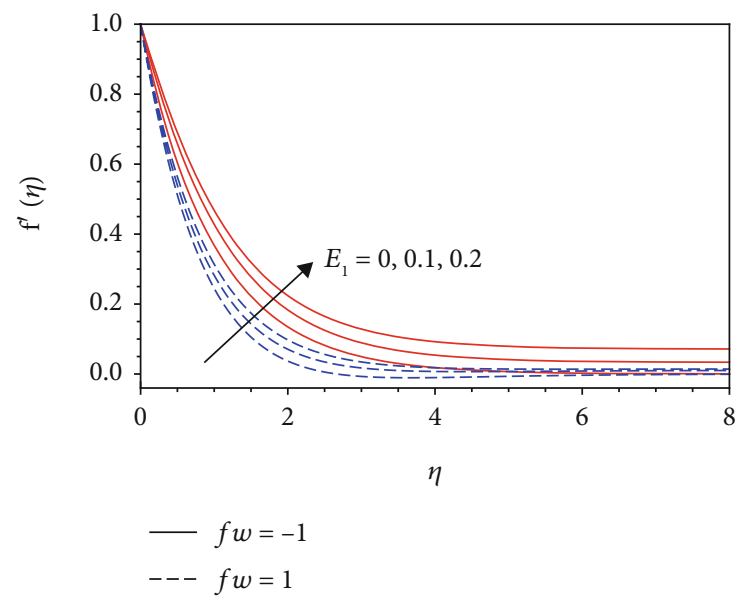

FIGURE 4: Velocity profile for different values of $E_{1}$ when $\mathrm{Rc}=0.5$, $M=1$, and $K_{p}=100$.

$$
\begin{aligned}
h_{2}{ }^{\prime}+h_{2}= & h_{1}{ }^{\prime}+h_{1}+C_{7}\left(h_{1}{ }^{\prime}+\operatorname{Sc}\left(f_{0} h_{1}{ }^{\prime}+f_{1} h_{0}{ }^{\prime}-f_{1}{ }^{\prime} h_{0}\right.\right. \\
& \left.\left.-f_{0}{ }^{\prime} h_{1}-\operatorname{Kc} h_{1}\right)\right)+C_{8}\left(h_{0}{ }^{\prime}+\operatorname{Sc}\left(f_{0} h_{0}{ }^{\prime}-f_{0}{ }^{\prime} h_{0}\right.\right. \\
& \left.\left.-\operatorname{Kch}_{0}\right)\right), h_{2}{ }^{\prime}(0)=0, h_{2}(\infty)=0 . .
\end{aligned}
$$

After solving the ODEs (60), (61), and (63) with the corresponding boundary conditions, we obtain

$$
\begin{aligned}
& h_{0}=e^{-\eta} . \\
& h_{1}=-C_{7} e^{-\eta}\left(-1+\mathrm{Sc}+f_{w} \mathrm{Sc}+\mathrm{KcSc}\right)(1+\eta) .
\end{aligned}
$$

We cannot write the third term $h_{2}$ here because of it being large. Hence, the solution $h\left(\eta, C_{i}\right)$ in terms of the parameters can be written as:

$$
h\left(\eta, C_{i}\right)=h_{0}(\eta)+h_{1}\left(\eta, C_{i}\right)+h_{2}\left(\eta, C_{i}\right), i=1,2,7,8 .
$$

For equation (65), we define its residual equation in the form

$$
R_{4}\left(\eta, C_{i}\right)=h^{\prime \prime}+\operatorname{Sc}\left(f h^{\prime}-f^{\prime} h-\mathrm{Kch}\right) .
$$

The unknown convergence parameters $C_{3}$ and $C_{4}$ can be optimally obtained from the following expressed conditions given below

$$
\begin{aligned}
\frac{\partial J_{4}\left(C_{i}\right)}{\partial C_{1}} & =\frac{\partial J_{4}\left(C_{i}\right)}{\partial C_{2}}=\frac{\partial J_{4}\left(C_{i}\right)}{\partial C_{7}}=\frac{\partial J_{4}\left(C_{i}\right)}{\partial C_{8}} \\
& =0 \text {, where } J_{4}\left(C_{i}\right)=\int_{0}^{0.1} R_{4}^{2}\left(\eta, C_{i}\right) d \eta .
\end{aligned}
$$

In the particular case when $\mathrm{Rc}=K_{p}=0.5, E_{1}=\mathrm{Sc}=0.1$, $f_{w}=\mathrm{Kc}=1, M=2$, one can obtain the results of the convergence parameters. Hence, the values are calculated as

$$
\begin{array}{ll}
C_{1}=0.14227123495837554, & C_{2}=-0.0985828777094099, \\
C_{7}=1.0547354705428924, & C_{8}=0.7632828804603283 .
\end{array}
$$

After substituting all the parameters, we get the approximate analytic solution as given below

$$
\begin{aligned}
h(\eta)= & e^{-\eta}+0.7383148293800247 e^{-\eta}(1+\eta)+e^{-3 \eta} \\
& \cdot\left(-0.0037514629487135163+0.16790779466661795 e^{\eta}\right. \\
& +0.17398430721442748 e^{2 \eta}+0.4985455077015227 e^{2 \eta} \eta \\
& \left.+0.2710538084617421 e^{2 \eta} \eta^{2}\right) .
\end{aligned}
$$




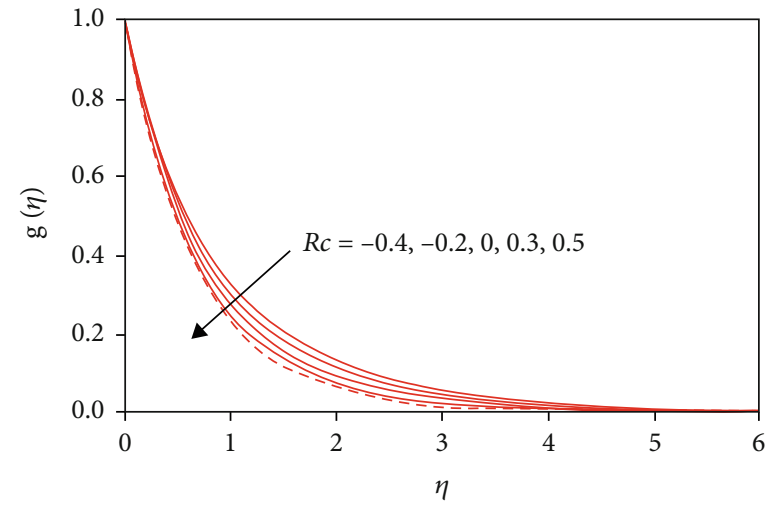

(a)

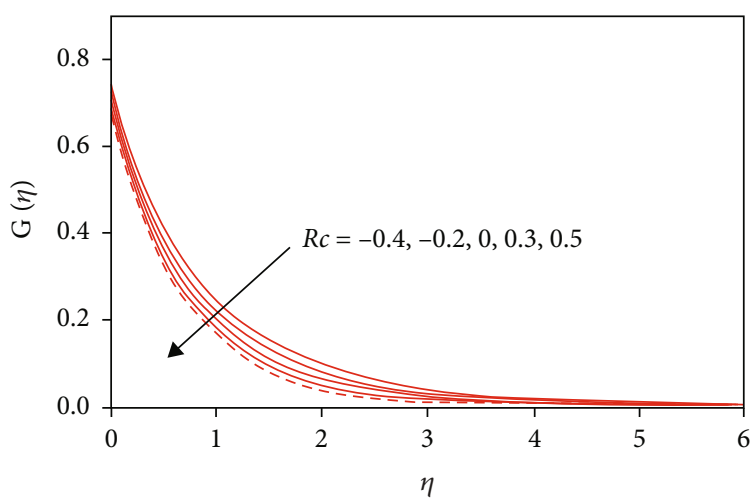

(b)

FIGURE 5: Temperature profiles for various values of $\operatorname{Rc}$ when $\operatorname{Pr}=3, M=\operatorname{Rd}=1, \mathrm{Ec}=E_{1}=0.1, Q=0$ and $f w=0$ : (a) $g$ in the PST case and (b) $G$ in the PHF case.

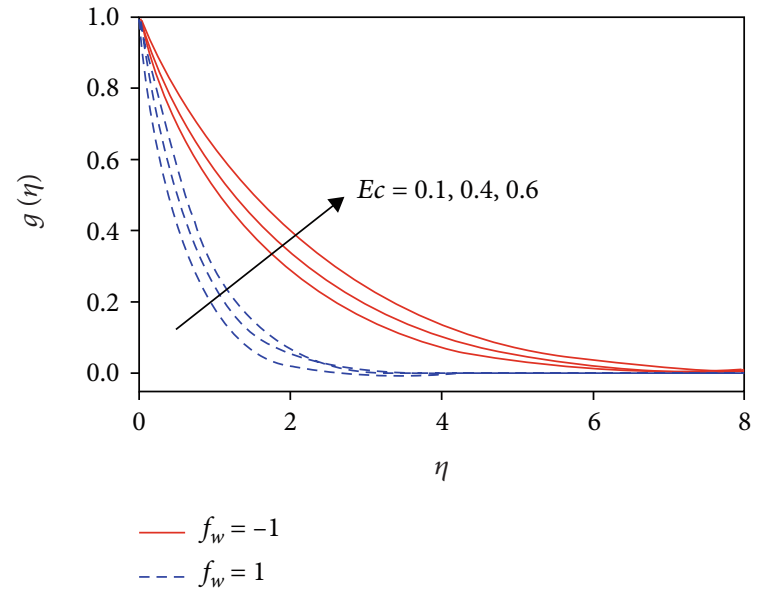

(a)

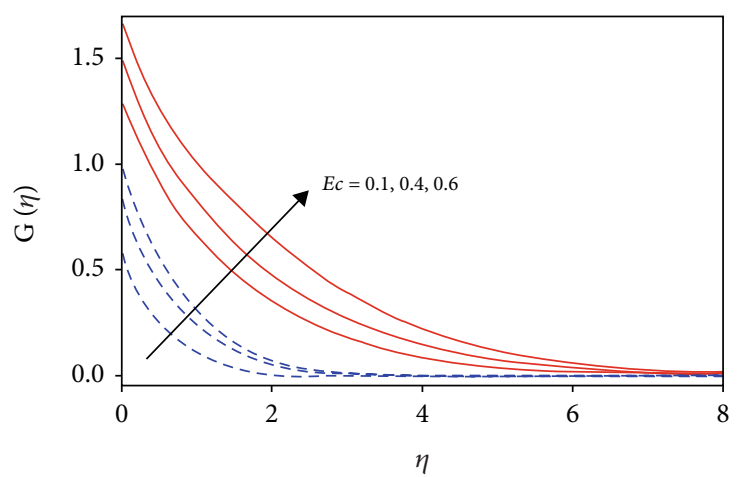

$f_{w}=-1$

$--f_{w}=1$

FIgURe 6: Temperature profiles for various values of $\mathrm{Ec}$ with $\operatorname{Pr}=2, M=1, \mathrm{Rc}=0.5, E_{1}=0.1, Q=0.2$ and $\mathrm{Rd}=1:$ (a) $g$ in the PST case and (b) $G$ in the PHF case.

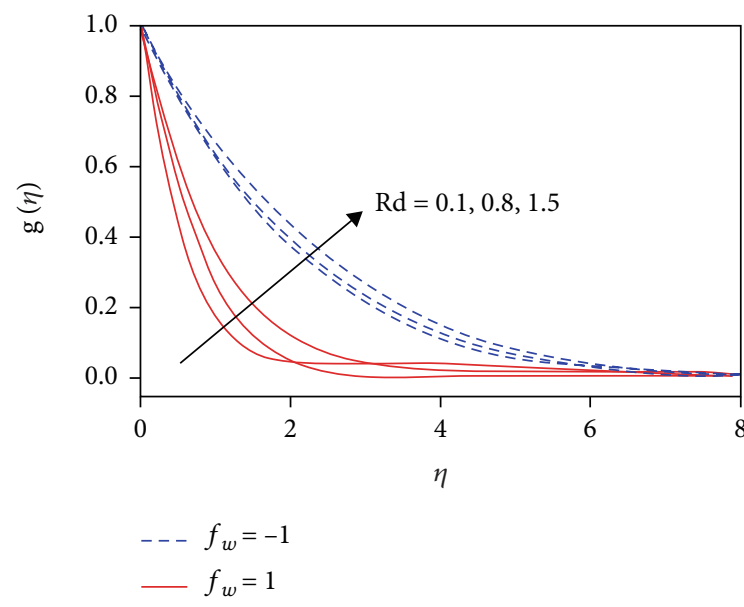

(a)

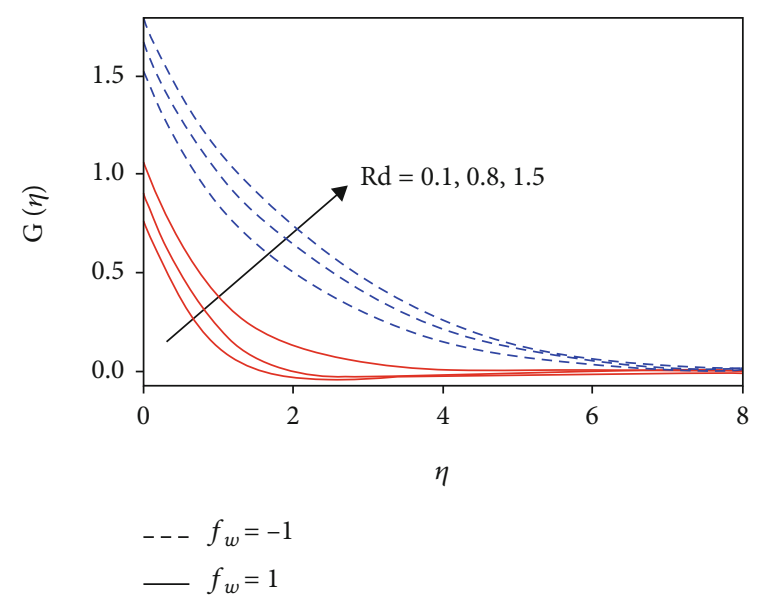

(b)

Figure 7: Temperature profiles for $\mathrm{Rc}=0.5, E_{1}=0.1, M=1, K_{p}=100, Q=0.2, \mathrm{Ec}=0.6$, and $\mathrm{Pr}=2$ : (a) PST case and (b) PHF case. 


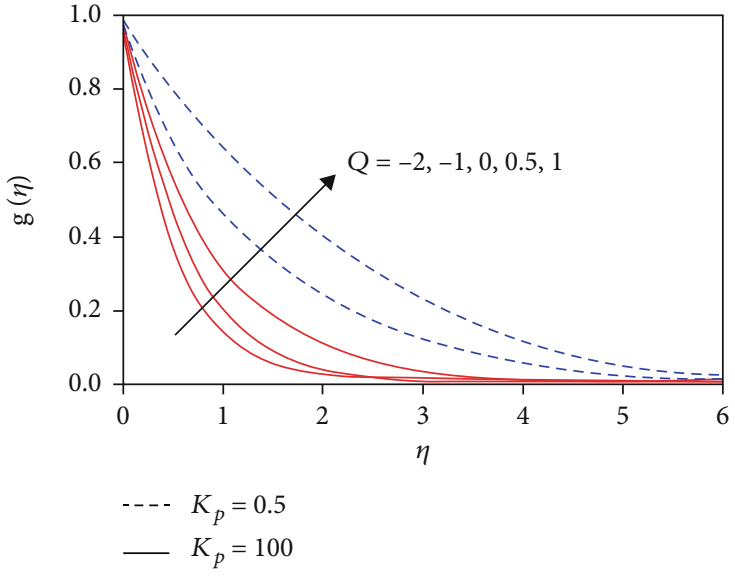

(a)

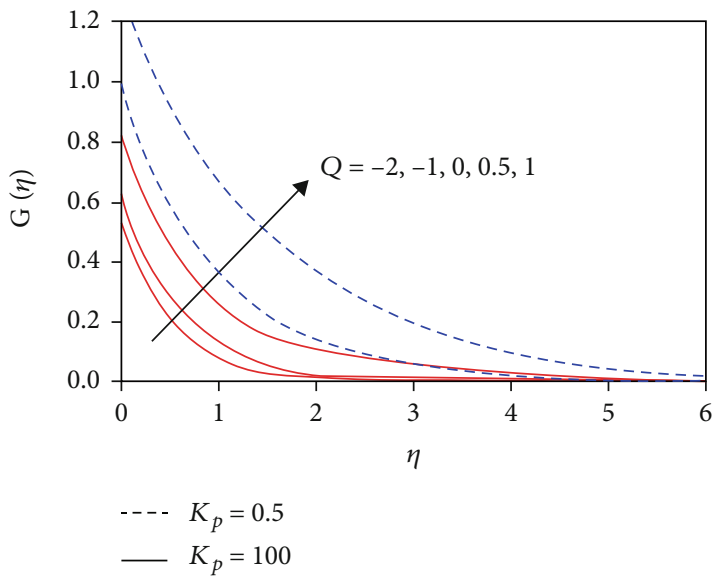

(b)

Figure 8: Temperature profiles for various values of $Q$ with $\operatorname{Pr}=2, M=\operatorname{Rd}=1, \mathrm{Ec}=E_{1}=0.1$ and $\mathrm{Rc}=0.5$, and $f w=0$ : (a) $g$ in the PST case and (b) $G$ in the PHF case.

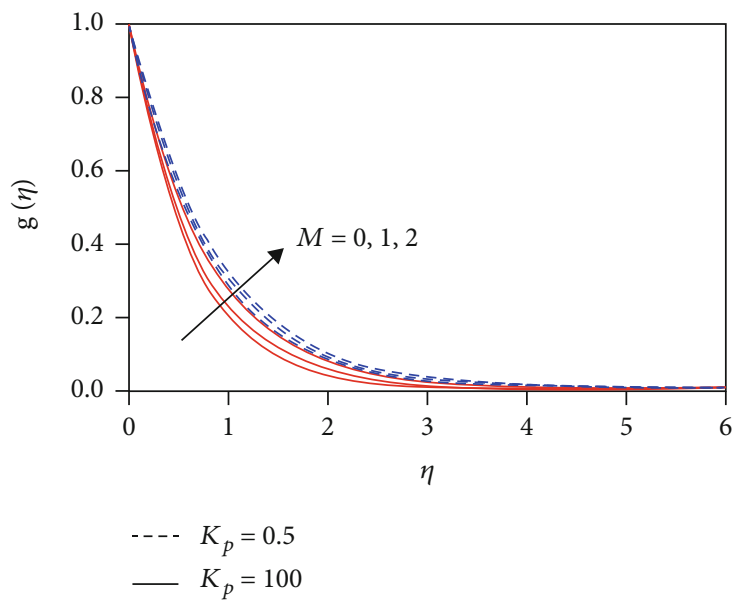

(a)

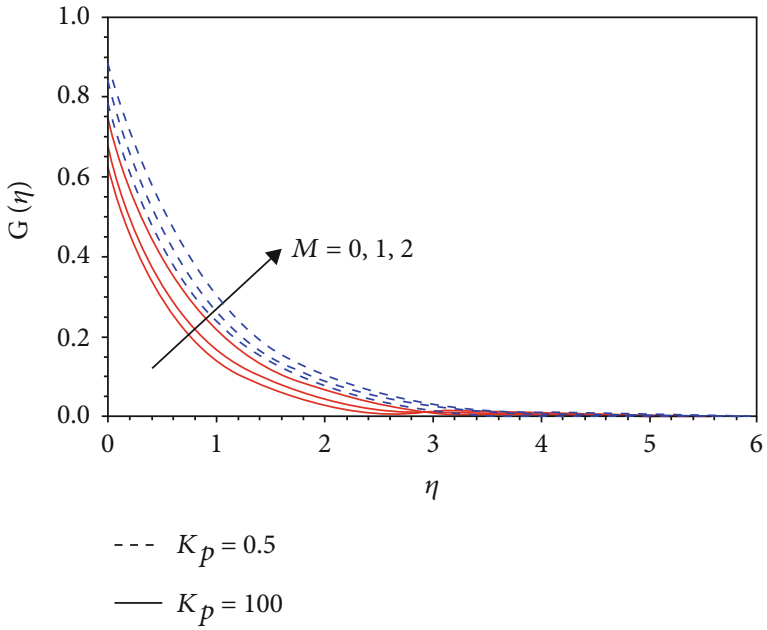

(b)

Figure 9: Temperature profiles for various values of $M$ when $\mathrm{Rc}=0.5, E_{1}=\mathrm{Ec}=0.1, f_{w}=0, \operatorname{Pr}=2$ and $Q=\mathrm{Rd}=0.2:(\mathrm{a}) \mathrm{g}$ in the PST case and (b) $G$ in the PHF case.

The local Sherwood number can be expressed as

$$
S h_{x} \operatorname{Re}_{x}^{-1 / 2}=-h^{\prime}(0)
$$

\section{Result and Discussion}

In the course of the discussion, the following aspects are highlighted:

(i) Effect of electric field and permeability of the medium on flow characteristics

(ii) Effect of diffusion species as well as the first-order chemical reaction

(iii) Relative response of two viscoelastic models to temperature and velocity distribution in the existence of uniform porous matrix (iv) The dimensionless ODEs (9), (27), (36), and (56) with the corresponding boundary conditions are solved by using the approximate analytic technique OHAM

It is important to note that $\mathrm{Rc}>0, \mathrm{Rc}<0, \mathrm{Rc}=0$ represent second grade, Walters $B^{\prime}$, and viscous fluids, respectively.

Figure 1 displays the velocity distribution of secondgrade fluid for suction $\left(f_{w}>0\right)$, injection $\left(f_{w}<0\right)$, and impermeable plate $\left(f_{w}=0\right)$ in the presence $\left(K_{p}=0.5\right)$ and absence $\left(K_{p}=100\right)$ of the porous matrix. The porous medium $\left(K_{p}=0.5\right)$ lessens the primary velocity at all points as a result of resistive force accessible by the porous medium which results in thinning of BL. Further, it is motivating to note that the suction at the plate decreases the velocity. Consequently, it is decided that the combined effect of porous 


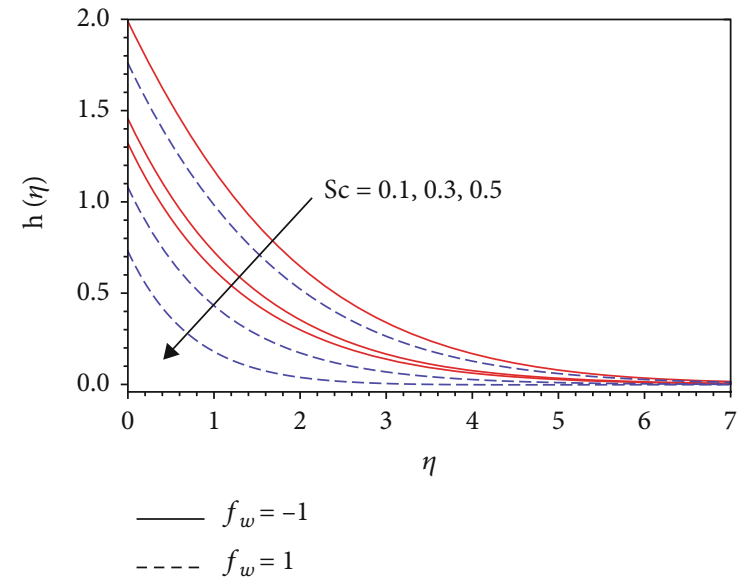

Figure 10: Concentration profile for various values of Sc when Rc $=0.5, E_{1}=0.1, M=2, \mathrm{Kc}=1$ and $K_{p}=100$.

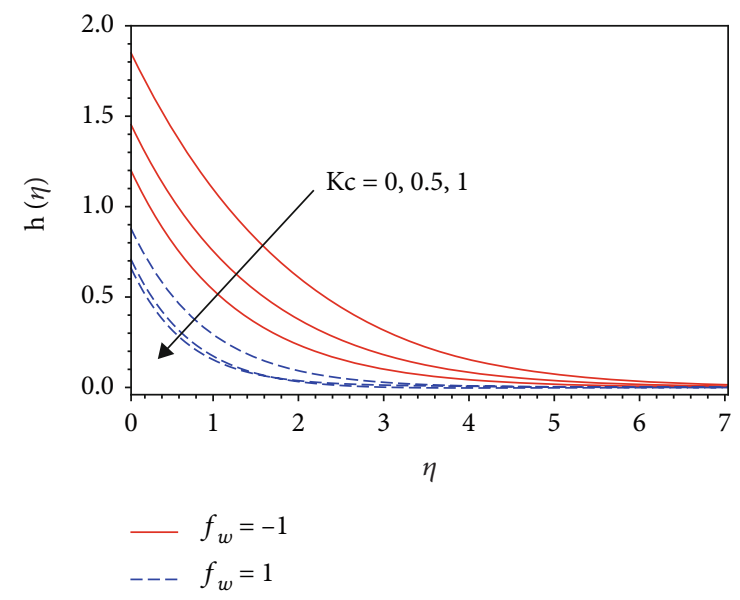

FIgURE 11: Concentration profile for various values of Kc when Rc $=0.5, E_{1}=0.1, M=2, \mathrm{Sc}=0.6$ and $K_{p}=100$.

matrix and suction is encouraging the thinning of $\mathrm{BL}$ which favors the stability of the flow.

Figure 2 displays the velocity profiles for Walters $B^{\prime}(\mathrm{Rc}$ $=-0.2)$ viscous $(R c=0)$ and second-grade flow $(R c=0.2)$. It is perceived that velocity achieves low value in the case of Walters' $B^{\prime}$ model, in the presence of a porous medium. Viscoelastic flows are disposed to instabilities as a result of nonlinearity in the constitutive equations. Instabilities are mainly driven by the fluid normal stresses (elasticity) or by the nature of the boundary conditions. Therefore, the elastic property of Walters' flow model in combination with the permeability of the porous medium reduces the BL thickness and hence decreases the instability.

The variation of the velocity field for various values of $M$ is illustrated in Figure 3. One can easily understand from the figure that the velocity significantly declines with a rise in the values of $M$ in the absence of an electric field $\left(\left(E_{1}=0\right)\right)$. It is clear that the magnetic field depends on the Lorentz force, which is stronger for a greater magnetic field. Due to the

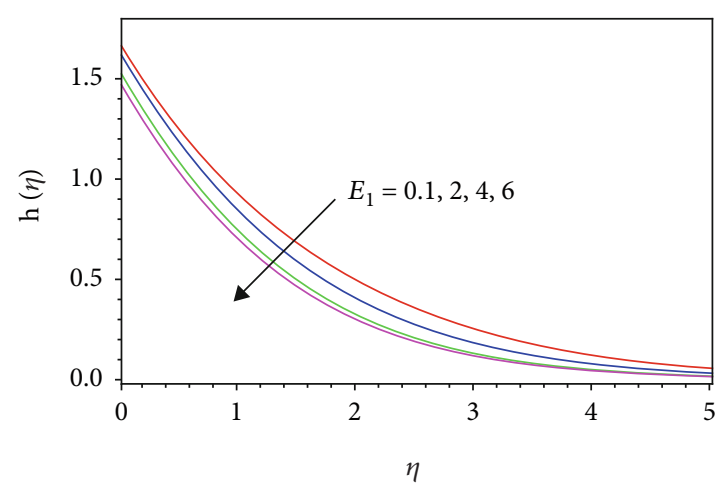

FIgURE 12: Concentration profile for various values of $E_{1}$ when Rc $=0.5, \mathrm{Kc}=1, f_{w}=0, M=2, \mathrm{Sc}=0.2$ and $K_{p}=100$.

TABLE 1: Comparison of the values of $f^{\prime \prime}(0)$ when $E_{1}=f_{w}=0$ and $K_{p}=100$.

\begin{tabular}{lccc}
\hline$M$ & Rc & Nayak et al. [33] & Present result \\
\hline 1 & -0.5 & -2.00499 & -2.00328365944 \\
0.5 & -0.5 & -1.73781 & -1.73779734058 \\
1 & 0.5 & -1.15758 & -1.14375034720 \\
1 & 1 & -1.00250 & -1.00098308049 \\
0.5 & 1 & -0.86891 & -0.86969982367 \\
0.5 & 0.5 & -1.00333 & -1.00284187010 \\
\hline
\end{tabular}

nonexistence of an electric field, the Lorentz force raises the frictional force, which acts as a delaying force that opposes the viscoelastic fluid flow.

The effect of $E_{1}$ on $f^{\prime}(\eta)$ is portrayed in Figure 4 . As the magnitudes of $E_{1}$ boost up, the velocity BL rises nearer to the stretching plate. For remarkable enhancement in the value of $E_{1}$, the struggle among the fluid particles rises, and hence, the Lorentz force inclines to speed up the body forces, and it leads to escalating in the flow velocity and thicker momentum boundary layer. The electric field has the same impact for the case of injection and suction as demonstrated in the figure.

Figures 5(a) and 5(b) display the temperature distribution in cases of PST and PHF cases, respectively, without suction/ injection. The effect of elasticity of the fluid subject to the present study on temperature distribution is opposite to that of the viscoelastic parameter that is the temperature declines at all points, and this is further contributed by the Walters $B^{\prime}$ model. Both the cases of PHF and PST show a similar effect.

Figures 6(a) and 6(b) demonstrate the effects of the Eckert number for second-grade fluid in the presence of suction/injection in both PST and PHF cases. The dissipation of energy of the flow pattern is measured by the Eckert number. It is seen that an increase in Ec increases the temperature and hence increases the thermal BL thickness. This leads to a reduction in the rate of heat transfer from the plate surface. 
The same effect is observed in the case of injection and suction.

Figures 7 (a) and 7(b) describe the effect of the radiation parameter in the case of second-grade fluid. It is perceived that an increase in Rd increases the temperature of the fluid layer and the processes get accelerated in both cases of suction and injection. The rise in temperature with an increase in radiation parameter causes growth in temperature gradient of the wall in both PST and PHF cases.

Figures 8(a) and 8(b) display the outcome of the internal heat generation/absorption parameter $Q$ on the temperature distribution $g(\eta)$ (PST) and $G(\eta)$ (PHF) in the case of second-grade fluid. This displays that a rise in heat source strength $(Q>0)$ increases the temperature. This is because of the generation of the heat in thermal BL which causes the temperature to escalate. In the same way, the heat sink causes temperature absorption resulting in a decline in temperature. The outcome holds good for both PHF and PST. The role of a porous matrix is to accelerate/decelerate the process in case of source/sink, respectively.

From Figures 9(a) and 9(b), it is observed that the impact of the magnetic field parameter $M$ is to raise the temperature profile with the increase in the magnetic field in the presence as well as in the absence of porous matrix. Physically, applying the magnetic field heats up the fluid and thus lessens the heat transfer rates from the wall causing rises in fluid temperature distributions.

Figure 10 exhibits the graph of concentration profiles for various values of Schmidt number Sc for the case of suction and injection. We observed that the concentration declines with a rise in the Schmidt number Sc for both cases. Thus, for higher values of the Schmidt number, the concentration of chemically reactive species is larger and lower for small values of Sc. Figure 11 displayed the influence of the chemical reaction parameter $\mathrm{Kc}$ for the case of suction and injection. The concentration profile $h(\eta)$ decreases with an increment of chemical reaction parameter. From Figure 12, we observed that the fluid concentration decreases for a large amount of an electric field parameter $E_{1}$. The rate of mass transfer at sheet increases because of an increment in the value of the electric field.

We examined the skin friction coefficient $f^{\prime \prime}(0)$ with the aid of a table. Table 1 is computed to validate the present analytic solution in a limiting case. It is observed that the present limiting results have a good match with the previously published results.

\section{Conclusions}

In this paper, we investigated the impact of electric field, heat source/sink, and chemical reaction on the MHD boundary layer flow of non-Newtonian fluid over a stretching surface. By using similarity transformations, the governing PDEs were converted into the dimensionless ordinary differential equations. We solved the transformed equations analytically using OHAM. The graphical illustrations of our results from the influence of relevant parameters on temperature, concentration, and velocity profiles are argued in depth. Some of the specific conclusions which have been derived from the study can be concluded as follows:

(i) The optimal homotopy asymptotic method is clear, effective, reliable, and efficient

(ii) Controlling and adjusting the convergence of the series solution using the convergence parameters are very simple

(iii) Porous matrix performing as an insulator to the vertical surface averts energy loss because of free convection which in turn improves the velocity

(iv) The existence of porous matrix and elasticity of the fluid overwhelms the resistive force of the magnetic field, and hence, the velocity rises due to the presence of both

(v) The occurrence of elasticity also leads to rising the temperature at all points regardless of the presence/absence of porous matrix

(vi) The slow rate of thermal diffusion in the existence of the magnetic field and porous matrix causes thinning of the thermal BL thickness

(vii) The variation in temperature is more sensitive on the account of heat flux

(viii) The existence of chemical reaction and porous matrix with moderate values of the magnetic parameter in case of heavier species lessens the concentration level

\section{Nomenclature}

$\begin{array}{ll}K_{p}: & \text { Permeability parameter } \\ M: & \text { Magnetic parameter } \\ B_{0}: & \text { Magnetic field strength } \\ Q: & \text { Heat source/sink parameter } \\ T_{0}: & \text { Temperature of the field } \\ P: & \text { Pressure nondimensional time } \\ q_{r}: & \text { Radiative heat flux } \\ \text { Rd: } & \text { Radiation parameter } \\ \text { Ec: } & \text { Eckert number } \\ C_{f}: & \text { Skin friction coefficient } \\ k_{0}: & \text { Modulus of the viscoelastic fluid } \\ m_{w}: & \text { Rate of mass flux } \\ K_{1}: & \text { Mean absorption coefficient } \\ k: & \text { Thermal conductivity } \\ \text { Rc: } & \text { Viscoelastic parameter } \\ \text { Pr: } & \text { Prandtl number } \\ \text { Sc: } & \text { Schmidt number } \\ T: & \text { Nondimensional temperature } \\ D: & \text { Molecular diffusivity } \\ C_{p}: & \text { Specific heat } \\ q_{w}: & \text { Wall heat flux } \\ E_{1}: & \text { Electric field parameter } \\ T_{1}: & \text { Temperature far from sheet } \\ T_{w}: & \text { Wall temperature }\end{array}$


Kc: $\quad$ Chemical reaction parameter

$A, B, E_{0}, E_{1}$ : Constants.

Greek Letters

$\alpha$ : Thermal diffusivity

$\sigma$ : Electrical conductivity

$v$ : Kinematics viscosity

$\rho$ : Density of the fluid

$\sigma^{*}$ : Stefan-Boltzmann constant

$\tau_{w}:$ Wall shear stress.

\section{Abbreviation}

BLF: $\quad$ Boundary layer flow

OHAM: Optimal homotopy asymptotic method

2D: $\quad$ Two dimensional

MHD: Magnetohydrodynamic

BC: $\quad$ Boundary condition

BL: $\quad$ Boundary layer

3D: $\quad$ Three dimensional

ODE: Ordinary differential equation.

\section{Data Availability}

No data support for this particular research.

\section{Conflicts of Interest}

The author declared no potential conflicts of interest with respect to the research, authorship, and/or publication of this article.

\section{References}

[1] B. C. Sakadis, "Boundary-layer behavior on continuous solid surfaces: II. The boundary layer on a continuous flat surface," AICHE Journal, vol. 7, no. 2, pp. 221-225, 1961.

[2] L. J. Crane, "Flow past a stretching plate," Zeitschrift für angewandte Mathematik und Physik ZAMP, vol. 21, no. 4, pp. 645647, 1970.

[3] A. G. Madaki, R. Roslan, R. Kandasamy, and M. S. H. Chowdhury, "Flow and heat transfer of nanofluid over a stretching sheet with non-linear velocity in the presence of thermal radiation and chemical reaction," AIP Conference Proceedings, vol. 1830, no. 1, pp. 020014-1-020014-10, 2017.

[4] L. Zheng, C. Zhang, X. Zhang, and J. Zhang, "Flow and radiation heat transfer of a nanofluid over a stretching sheet with velocity slip and temperature jump in porous medium," Journal of the Franklin Institute, vol. 350, no. 5, pp. 990-1007, 2013.

[5] H. Dessie and N. Kishan, "MHD effects on heat transfer over stretching sheet embedded in porous medium with variable viscosity, viscous dissipation and heat source/sink," Ain Shams Engineering Journal, vol. 5, no. 3, pp. 967-977, 2014.

[6] D. Pal, "Heat and mass transfer in stagnation-point flow towards a stretching surface in the presence of buoyancy force and thermal radiation," Meccanica, vol. 44, no. 2, pp. 145-158, 2009.

[7] S. K. Khan, "Heat transfer in a viscoelastic fluid flow over a stretching surface with heat source/sink, suction/blowing and radiation," International Journal of Heat and Mass Transfer, vol. 49, no. 3-4, pp. 628-639, 2006.

[8] M. Madhu and N. Kishan, "Finite element analysis of MHD viscoelastic nanofluid flow over a stretching sheet with radiation," Procedia Engineering, vol. 127, pp. 432-439, 2015.

[9] M. Babaelahi, G. Domairry, and A. A. Joneidi, "Viscoelastic MHD flow boundary layer over a stretching surface with viscous and ohmic dissipations," Meccanica, vol. 45, no. 6, pp. 817-827, 2010.

[10] B. Raftari and K. Vajravelu, "Homotopy analysis method for MHD viscoelastic fluid flow and heat transfer in a channel with a stretching wall," Communications in Nonlinear Science and Numerical Simulation, vol. 17, no. 11, pp. 4149-4162, 2012.

[11] M. Goyal and R. Bhargava, "Numerical solution of MHD viscoelastic nanofluid flow over a stretching sheet with partial slip and heat source/sink," ISRN Nanotechnology, vol. 2013, Article ID 931021, 11 pages, 2013.

[12] M. Madhu and N. Kishan, "Boundary layer flow of viscoelastic nanofluid over a wedge in the presence of buoyancy force effects," Computational Thermal Sciences: An International Journal, vol. 9, no. 3, pp. 257-267, 2017.

[13] G. Aliy and N. Kishan, "Electrical MHD viscoelastic nanofluid flow and heat transfer over a stretching sheet with convective boundary condition. Optimal homotopy asymptotic method analysis," Journal of Nanofluids, vol. 8, no. 2, pp. 317-326, 2019.

[14] A. B. Jafar, S. Shafie, and I. Ullah, "Magnetohydrodynamic boundary layer flow of a viscoelastic fluid past a nonlinear stretching sheet in the presence of viscous dissipation effect," Coatings, vol. 9, no. 8, p. 490, 2019.

[15] J. R. Konda, N. P. Madhusudhana Reddy, R. Konijeti, and A. Dasore, "Effect of non-uniform heat source/sink on MHD boundary layer flow and melting heat transfer of Williamson nanofluid in porous medium," Multidiscipline Modeling in Materials and Structures, vol. 15, no. 2, pp. 452-472, 2019.

[16] T. Hayat, M. Awais, A. Safdar, and A. A. Hendi, "Unsteady three dimensional flow of couple stress fluid over a stretching surface with chemical reaction," Nonlinear Analysis: Modelling and Control, vol. 17, no. 1, pp. 47-59, 2012.

[17] B. J. Gireesha, G. K. Ramesh, M. S. Abel, and C. S. Bagewadi, "Boundary layer flow and heat transfer of a dusty fluid flow over a stretching sheet with non-uniform heat source/sink," International Journal of Numerical Methods for Heat and Fluid Flow, vol. 37, no. 8, pp. 977-982, 2011.

[18] A. B. Parsa, M. M. Rashidi, and T. Hayat, "MHD boundarylayer flow over a stretching surface with internal heat generation or absorption," Heat Transfer-Asian Research, vol. 42, no. 6, pp. 500-514, 2013.

[19] P. Weidman, "Comment on "Heat transfer in MHD viscoelastic boundary layer flow over a stretching sheet with thermal radiation and non-uniform heat source/sink", M.M. Nandeppanavar, K. Vajravelu \& M.S. Abel [Commun Nonlinear Sci Numer Simulat 16 (2011) 3578-3590]," Communications in Nonlinear Science and Numerical Simulation, vol. 19, no. 9, pp. 3412-3417, 2014.

[20] M. S. Abel and M. M. Nandeppanavar, "Heat transfer in MHD viscoelastic boundary layer flow over a stretching sheet with non-uniform heat source/sink," Communications in Nonlinear Science and Numerical Simulation, vol. 14, no. 5, pp. 2120 2131, 2009. 
[21] A. G. Madaki, R. Roslan, R. Kandasamy, and I. Hashim, "Flow and heat transfer of nanofluid over a dynamic stretching sheet with nonlinear velocity and thermal radiation," Journal of Engineering and Applied Science, vol. 12, pp. 5136-5146, 2017.

[22] V. Marinca, N. Herişanu, C. Bota, and B. Marinca, "An optimal homotopy asymptotic method applied to the steady flow of a fourth-grade fluid past a porous plate," Applied Mathematics Letters, vol. 22, no. 2, pp. 245-251, 2009.

[23] V. Marinca and N. Herisanu, "The optimal homotopy asymptotic method for solving Blasius equation," Applied Mathematics and Computation, vol. 231, pp. 134-139, 2014.

[24] V. Marinca and N. Herisanu, "On the flow of a Walters-type B' viscoelastic fluid in a vertical channel with porous wall," International Journal of Heat and Mass Transfer, vol. 79, pp. 146165, 2014.

[25] V. Marinca and N. Herisanu, "An optimal homotopy asymptotic approach applied to nonlinear MHD Jeffery-Hamel flow," Mathematical Problems in Engineering, vol. 2011, Article ID 169056, 16 pages, 2011.

[26] F. Mabood, W. A. Khan, and A. I. Ismail, "Optimal homotopy asymptotic method for flow and heat transfer of a viscoelastic fluid in an axisymmetric channel with a porous wall," PLoS One, vol. 8, no. 12, p. e83581, 2013.

[27] M. Mustafa, "Viscoelastic flow and heat transfer over a nonlinearly stretching sheet: OHAM solution," Journal of Applied Fluid Mechanics, vol. 9, pp. 1321-1328, 2016.

[28] M. S. Abdel-Wahed, E. M. A. Elbashbeshy, and T. G. Emam, "Flow and heat transfer over a moving surface with nonlinear velocity and variable thickness in a nanofluids in the presence of Brownian motion," Applied Mathematics and Computation, vol. 254, pp. 49-62, 2015.

[29] A. Gizachew, "Analytical solutions of an MHD heat and mass transfer of a Jeffery fluid flow over a stretching sheet with the effect of slip velocity," Advances in Applied Sciences, vol. 3, no. 3 , p. $34,2018$.

[30] G. Aliy and N. Kishan, "Optimal homotopy asymptotic solution for cross-diffusion effects on slip flow and heat transfer of electrical MHD non-Newtonian fluid over a slendering stretching sheet," International Journal of Applied and Computational Mathematics, vol. 5, no. 3, p. 80, 2019.

[31] G. Aliy and N. Kishan, "Optimal homotopy asymptotic solution for thermal radiation and chemical reaction effects on electrical MHD Jeffrey fluid flow over a stretching sheet through porous media with heat source," Applications and Applied Mathematics: An International Journal (AAM), no. 4, pp. 150-175, 2019.

[32] M. A. Khan, N. H. M. Ali, and S. Ullah, "Application of optimal homotopy asymptotic method for Lane-Emden and Emden-Fowler initial and boundary value problems," Proceedings of the International Conference on Mathematical Sciences and Technology 2018 (MATHTECH2018), vol. 2184, no. 1, pp. 060022-1-060022-6, 2019.

[33] M. K. Nayak, G. C. Dash, and L. P. Singh, "Heat and mass transfer effects on MHD viscoelastic fluid over a stretching sheet through porous medium in presence of chemical reaction," Propulsion and Power Research, vol. 5, no. 1, pp. 7080, 2016. 